\title{
Correction to: An overview of structural equation modeling: its beginnings, historical development, usefulness and controversies in the social sciences
}

\section{Piotr Tarka ${ }^{1}$}

Published online: 21 February 2022

(c) The Author(s) 2022

\section{Correction to: Qual Quant (2018) 52:313-354 https://doi.org/10.1007/s11135-017-0469-8}

The author inadvertently failed to attribute a portion of text in their manuscript to [1]. While [1] is referenced throughout this article, there are some near direct quotations which have not been acknowledged. The affected sections are listed below.

1. Spearman (1904) is often cited in the literature as "the founding father of factor analysis, even though one year earlier Pearson (1901a) published a paper on fitting planes by orthogonal least squares, [which was] the foundation for principal component analysis [that was also] applied to the analysis of correlation matrices by Hotelling (1933)" [1].

2. Thurstone "noted that a vanishing tetrad difference [implies] a vanishing second-order determinant of the matrix of [observable variables], and [therefore decided to extend it to] the vanishing of higher-order determinants as a condition for more than one factor. [Later] he generalized the result [as] the number of common factors [that was determined] by the rank of the matrix of observables (Harman 1960). [Next,] Thurstone (1935) developed the centroid method of factoring a correlation matrix (as a pragmatic compromise to the computationally-burdensome principle axis method). Moreover, he developed a definition of [a] simple structure for factor analysis based on five principles (the most important of which [was] to minimize negative loadings and maximize zero loadings) to facilitate interpretation and [to] insure that [the] loadings were invariant to the inclusion of other items" [1].

3. What truly made Wright develop path analysis was the fact that he was dissatisfied with the results of the partial correlation analysis that was being conducted "which remained

The original article can be found online at https://doi.org/10.1007/s11135-017-0469-8.

Piotr Tarka

piotr.tarka@ue.poznan.pl

1 Department of Market Research, Poznan University of Economics, al. Niepodleglosci 10, 61-875 Poznan, Poland 
far from a causal explanation. Consequently, [he] developed path analysis to impose a causal structure, with structural coefficients, on the observed correlations" [1].

4. These scholars made a milestone in providing an understanding of the principles of SEM by defining the 'structural relation' as 'the theoretical relation postulated a priori' in a single-equation multivariate linear model in which the partial regression coefficient represented a 'structural coefficient' [1]. Frisch (1934) was, however, sceptic of the "use of probability models for economic data, which were rarely the result of a sampling process, and of OLS [(Ordinary Least Squares)] regression, because measurement errors existed not only [in the] dependent variables but [also in the] independent variables" [1]. Frisch treated observable variables as fallible indicators of the latent variables to distinguish 'true relations' from 'confluent relations' [1]. Haavelmo (1943, 1944), on the other hand, contributed to the development of SEM by "specifying a probability model for econometric models [and concisely described] the Neyman-Pearson (1933) approach to hypothesis testing [by] using the probability approach for estimation, testing, and forecasting. He also distinguished between two models of the source of stochastic components, [i.e.,] errors-in-variables models, as emphasized by Frisch (1934), and random [shock] models, [as] introduced by Slutsky (1937). This framework is often [defined] as the "probabilistic revolution"' in econometrics and has had a lasting impact on the field, particularly in cementing the Neyman-Pearson approach to inference over others, such as Bayesian approaches" [1]. Finally, Haavelmo (1943, 1944) advanced SEM by proving that "OLS estimates are biased in a two-equation supplydemand model [and distinguished] between [the structure for] equations and what Mann and Wald (1943) termed [as] the reduced-form equation" [1]. He applied the maximum likelihood (ML) estimation to the system of equations, showing its equivalence to OLS when applied to the reduced form, and further specified the necessary conditions for identification in terms of partial derivatives of the likelihood function (Matsueda 2012). Later, Koopmans et al. (1950), who also worked in the 'Cowles Commission',2 helped to solve "major problems of identification, estimation, and testing of [SEM] models" [1].

5. Later, "in 1975, Duncan authored an excellent [...] text for path analysis and structural equation models in which he echoed Frisch and Haavalmo's concept of autonomy"the structural form is that [of] parameterization [...] in which the coefficients are (relatively) unmixed, invariant, and autonomous"' (Duncan 1975; p. 151). He also distinguished [between] forms of social change from trivial changes in sampling or exogenous variables ([which] leave the structural coefficients intact), to deeper changes in the structural coefficients (which provide [an understanding for the explanation of SEM] models) and changes in the model's structure itself, and provided [important hints] for applying [the] structural models" [1].

6. Simultaneously, work was continued "on factor models for dichotomous variables, [e.g.,] Bock and Lieberman (1970) [...] used tetrachoric correlations and an ML estimator for a single factor model, and Christofferson (1975) [...] generalized this to multiple factors using a GLS estimator (see also Muthén 1978). Muthén (1979) subsequently developed a multiple-indicator structural probit model, [while] Winship and Mare (1983) [showed how to apply] multivariate probit models estimated by ML to multiple-indicator structural equation models and path analysis" [1].

7. Glymour et al. (1987), "instead of focusing on the estimation and testing of structural models specified a priori, [worked on computer algorithms (TETRAD) derived] from artificial intelligence [in order] to 'discover' [the] causal structure. [Thus they returned] to the earlier ideas of Spearman, Frisch, Simon, Blalock, and Costner, who 
tried, in various ways, to induce [a] causal structure from patterns of association among variables" [1].

8. "Matsueda and Bielby (1986) and Satorra and Saris (1985) independently showed how to calculate the power of the likelihood ratio test in covariance structure models [by] using the non-central [Chi-square] distribution and [...] presented a nearly identical way of approximating the non-centrality parameter. They [proved] that the likelihood ratio test statistic is asymptotically equivalent to a quadratic form" [1].

Open Access This article is licensed under a Creative Commons Attribution 4.0 International License, which permits use, sharing, adaptation, distribution and reproduction in any medium or format, as long as you give appropriate credit to the original author(s) and the source, provide a link to the Creative Commons licence, and indicate if changes were made. The images or other third party material in this article are included in the article's Creative Commons licence, unless indicated otherwise in a credit line to the material. If material is not included in the article's Creative Commons licence and your intended use is not permitted by statutory regulation or exceeds the permitted use, you will need to obtain permission directly from the copyright holder. To view a copy of this licence, visit http://creativecommons.org/licenses/by/4.0/.

\section{Reference}

[1] Matsueda, R.L.: Key advances in the history of structural equation modeling. In: Hoyle, R.H. (ed.) Handbook of Structural Equation Modeling, pp. 17-42. The Guilford Press (2012)

Publisher's Note Springer Nature remains neutral with regard to jurisdictional claims in published maps and institutional affiliations. 